\title{
Methods Comparison for the Synthesis of Deca-dodecasil 3 Rhombohedral (DDR3) Zeolite Crystals
}

\author{
Muhammad Mubashir, Yeong Yin Fong ", Lau Kok Keong,
}

Azmi Bin Mohd Shariff

\author{
Department of Chemical Engineering, Universiti Teknologi PETRONAS, Bandar Seri Iskandar \\ 31750 Perak, Malaysia \\ ${ }^{*}$ Corresponding author. Tel.: +605-3687564; fax: +605-3656176. \\ Email address: yinfong.yeong@petronas.com.my
}

Key words: DDR3 zeolite Crystals, Synthesis, Sonication

\begin{abstract}
In the present work, DDR3 zeolite crystals were synthesized using two different methods. The silica sources used to synthesize DDR3 crystals were tetramethoxysilane (TMOS) and Ludox40. The resultant samples were characterized using X-ray Diffraction (XRD) and Field Emission Scanning Electron Microscope (FESEM). The XRD results showed that the peaks representing DDR3 structure were not obtained for the sample synthesized in 5 days at room temperature with ultrasonic pre-treatment of $3 \mathrm{~h}$ using Ludox-40 as silica source. On the other hand, the XRD pattern obtained for the sample synthesized in 25 days at $160^{\circ} \mathrm{C}$ using TMOS as a silica source were similar with the XRD peaks reported in the literature. From these results, it can be concluded that the synthesis conditions of 25 days at $160^{\circ} \mathrm{C}$ using TMOS as silica source were the favorable conditions in obtaining DDR3 crystal structure.
\end{abstract}

\section{Introduction}

Worldwide consumption of natural gas is increasing day by day, which reaches over 3.1 trillion cubic meters per year [1-2]. It is projected that the utilization of natural gas will be increased to 185 trillion cubic feet in 2040 [1]. Further, the presence of $\mathrm{CO}_{2}$ in natural gas drops the heating value and also causes pipeline corrosion [3]. Thus, different technologies are introduced to capture $\mathrm{CO}_{2}$ from natural gas including adsorption, absorption, cryogenic distillation and membranes [4]. Membranes are environmental friendly, simple, easy to handle and occupy less space [3]. Polymeric membranes have limited uses in chemical industries because of their low thermal stability and low separation performance compared to inorganic membranes [5]. Recently, inorganic membranes have been extensively studied because of their advantages including; high thermal and chemical stability and high selectivity [6]. Zeolite membranes are the potential membrane material for $\mathrm{CO}_{2}$ separation from natural gas because of their characteristic such as molecular sieving property. Researchers are widely conducting studies on the preparation of zeolite films since the last two decades. Zeolite membranes consist of ultra large, large, medium and small pore framework. DDR3 membrane is a type of zeolite membranes having small pores $(0.36 \times 0.44 \mathrm{~nm})$ framework and exhibited the highest selectivity in $\mathrm{CO}_{2}$ separation from natural gas [7].

DDR3 is a pure silica zeolite with a chemical formula of $\left(\mathrm{C}_{10} \mathrm{H}_{17} \mathrm{~N}\right)_{6}\left(\mathrm{~N}_{2}\right)_{9}\left[\mathrm{Si}_{120} \mathrm{O}_{240}\right]$. The crystal structure contains $4,5,6$, and $8 \mathrm{Si}$ atoms in rings. These rings are formed by combining decade hadron with dodeca hadron cages, resulting 19 hadron cage [8]. Figure 1 shows cage structure of decade hadron (a), dodeca hadron (b) and (c) 19 hadron [9]. 


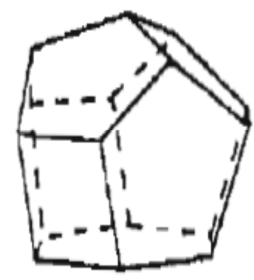

(a)

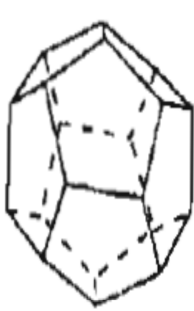

(b)

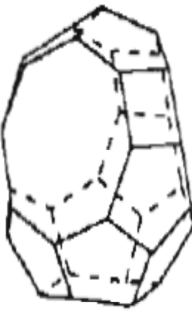

(c)

Fig 1. Cage structure of Decade hadron (a), dodeca hadron (b) and (c) 19 hadron [9].

In 1986, Gies. H [10] reported the synthesis of DDR3 zeolite particles for the first time. In 1994, den Exter et al. [11] optimized the synthesis duration, water concentration and conventional heating temperature for obtaining DDR3 zeolite crystals. They successfully synthesized DDR3 crystals in 25 days at $160{ }^{\circ} \mathrm{C}$ using tetramethoxysilane (TMOS). Subsequently, Tomita et al., 2004 [12] reported the synthesis of DDR3 zeolite particles following the method reported by den Exter et al. [11]. In 2007, Alves [9] studied the effect of water concentration, $\mathrm{pH}$ and aging time on the synthesis of DDR3 zeolite. In 2009, Qi-Liang et al.[13] synthesized DDR3 crystals in 9 days using fluoride medium in order to study the effect of synthesis conditions on the crystals size. In 2014, Mitali Sen et al. [14] successfully synthesized DDR3 crystals in 5 days at room temperature by applying ultrasonic pre-treatment on the synthesis solution for $3 \mathrm{~h}$ and Ludox-40 as silica source prior to the crystallization of DDR3 particles.

From the literature, although two common methods have been applied in the synthesis of zeolite membranes, including i) insitu hydrothermal growth and ii) secondary growth, the successful cases of the synthesis of DDR3 membranes have been reported by using secondary growth method. The critical issues relating to the secondary growth method include the preparation of colloidal zeolite seed suspension, $\mathrm{pH}$ of seed suspension, the effective seed coating on the support surface, and the identification of the secondary growth conditions. Therefore, a facile method to reduce the synthesis duration of DDR3 crystal prior to membrane formation is very crucial in order to reduce the overall synthesis duration of DDR3 membrane. Therefore, in the present work, two different methods will be used for the synthesis of DDR 3 crystals, as an initial stage for the investigation of possible time reduction in the synthesis of DDR3 crystal. The resulting DDR3 crystals were characterized for its crystallinity and morphology using XRD and FESEM.

\section{Experimental}

\subsection{Chemicals and Materials}

The chemical reagents used are colloidal Ludox-40 (Sigma Aldrich), tetramethoxysilane (Merck), structure directing agent (SDA) 1-adamantaneamine (Fisher Scientific $>96 \%$ ), ethylenediamine (Merck) for maintaining $\mathrm{pH}$ and di-ionized water.

\subsection{Synthesis of DDR3 Crystals}

DDR3 crystals were prepared by using conventional heating method described by den Exter et al. [11]. The mixture was synthesized by dissolving $1.422 \mathrm{~g}$ of 1 -adamantaneamine (Fisher Scientific $96 \%$ ) in $4.85 \mathrm{~g}$ of ethylenediamine (Merck) and then $40.464 \mathrm{~g}$ of di-ionized water was added rapidly. Then mixture was agitated at $200 \mathrm{rpm}$ for $1 \mathrm{~h}$ at room temperature. After heating the mixture for $1 \mathrm{hr}$ at $95{ }^{\circ} \mathrm{C}$ with continuous agitation, it was cooled down in ice for $20 \mathrm{~min}$. Then, $3.044 \mathrm{~g}$ of tetramethoxysilane (Merck) was added drop by drop in ice cooled mixture. Then mixture was again heated at for $1 \mathrm{~h}$ at $95^{\circ} \mathrm{C}$ with continuous agitation. Final mixture has molar ratio of 1- 
adamantaneamine: Silica: Ethylenediamine: Water $=47: 100: 404: 11,240$. The mixture was then heated in pressure vessel for 25 days at $160{ }^{\circ} \mathrm{C}$. The powder product was recovered by using centrifugation and washed with deionized water and then dried for overnight at $100{ }^{\circ} \mathrm{C}$. For comparison, DDR3 samples were also prepared by using the method reported by Mitali Sen et al. [14]. According to this method, two reactant mixtures were prepared simultaneously. Mixture 1 was prepared by adding $17.1 \mathrm{~g}$ of de-ionized water in $3.004 \mathrm{~g}$ of colloidal silica (Ludox-40) and mixture 2 was prepared by dissolving 1-adamantaneamine (Fisher Scientific 96\%) of $1.51 \mathrm{~g}$ and $4.808 \mathrm{~g}$ of ethylenediamine (Merck) in $17.1 \mathrm{~g}$ of water. Both mixtures were stirred separately at room temperature for $1 \mathrm{~h}$. After stirring, mixture 2 was added into mixture 1 and stirrer continuously for 1 $\mathrm{h}$. Then, the resulting mixture was sonicated for $3 \mathrm{~h}$ at room temperature. The molar composition of the solution was 0.51 -adamantaneamine: 4 ethylenediamine: 1 silica: 100 water. Then the solution was kept for aging for 5 days at room temperature for the crystal growth. The powder sample was collected following the above-mentioned procedure.

\section{Results and Discussion}

Both samples synthesized using different methods were characterized using X-ray diffraction. Figure 2 shows the comparison between the XRD patterns of the samples synthesized in 25 days at $160{ }^{\circ} \mathrm{C}$ without ultrasonic pre-treatment using tetramethoxysilane (TMOS) (Sample A) and sample synthesized in 5 days at room temperature with ultrasonic pre-treatment of $3 \mathrm{~h}$ using Ludox-40 (Sample B). Referring to Figure 2, the presence of peaks in the XRD pattern of sample A confirmed the crystalline structure of DDR3. The significant peaks at $2 \theta$ value are inconsistent with those peaks reported in the literature for DDR3 structure. However, as shown in Figure 2, sample B shows amorphous structure due to the absence of major peaks representing crystalline DDR3 structure. From these results, it can be confirmed that crystals synthesized in 25 days at $160{ }^{\circ} \mathrm{C}$ without ultrasonic pre-treatment demonstrated the structure of DDR3 topology.

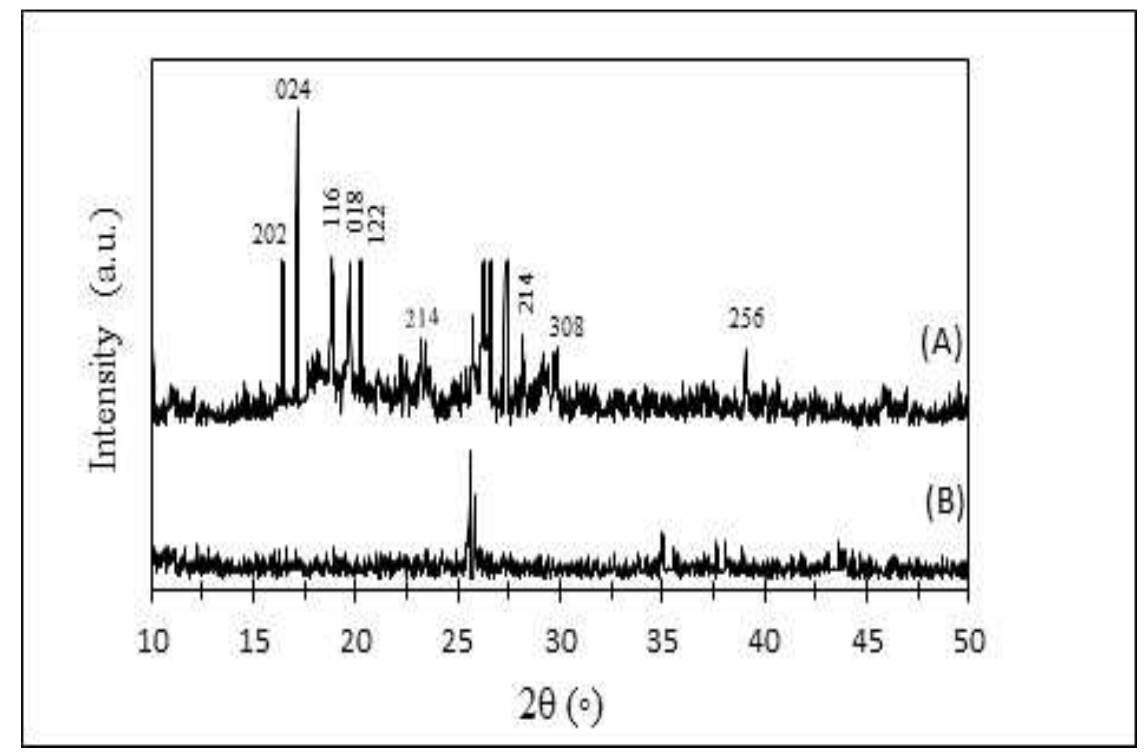

Fig 2. XRD pattern of DDR crystals synthesized for (A) 25 days and (B) 5 days.

Both samples (A \& B) were also characterized by using Field Emission Scanning Electron Microscope (FESEM). Figure 3 compares the morphology of sample (A) and sample (B). Referring Figure 3, sample (A) shows well-formed crystal morphology with narrow size distribution and mean crystals radius of $199 \mathrm{~nm}$. However, the morphology of sample (B) are unclear and amorphous phases were found. Therefore, it can be concluded that DDR3 crystals was successfully synthesized in 25 days at $160{ }^{\circ} \mathrm{C}$ using tetramethoxysilane (TMOS). 

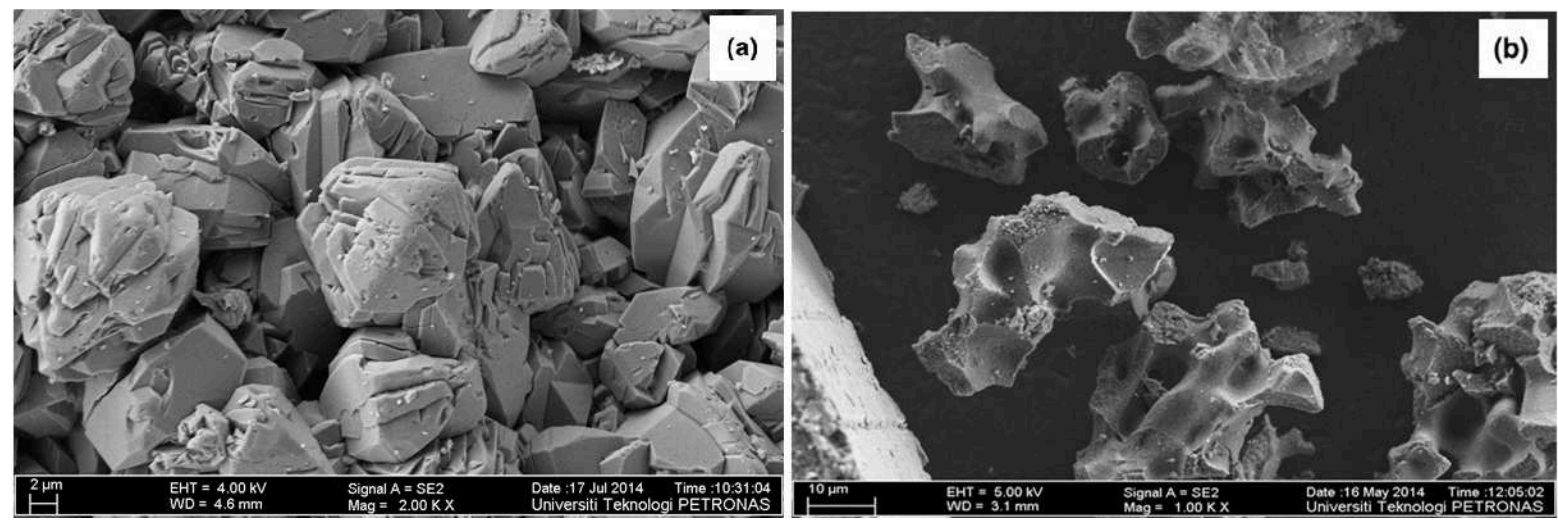

Fig 3. Field-emission scanning electron microscopy micrographs of samples synthesized in (a) 25 days at $160{ }^{\circ} \mathrm{C}$ and (b) 5 days at room temperature with ultrasonic pretreatment.

\section{Conclusions}

1. In this work, we concluded that hydrothermal growth synthesis temperature is a preferable condition for synthesis of DDR3 zeolite crystals.

2. XRD peaks verified that the DDR3 crystals can be synthesized in 25 days at $160{ }^{\circ} \mathrm{C}$ without ultrasonic pre-treatment of synthesis sol.

3. The ways to reduce the synthesis duration of DDR3 crystals still remain a challenging issue. Ultrasonic pre-treatment is a potential method in reducing the crystallization duration of DDR3 crystal. Therefore, future works need to be focused on the variation of synthesis parameters for better comparison. For example, using same silica source and increase the hydrothermal growth duration, e.g. 10 days after ultrasonic pretreatment.

\section{Acknowledgements}

The financial and technical supports provided by $\mathrm{CO}_{2}$ Management (MOR) research group, Universiti Teknologi PETRONAS and Ministry of Education (Higher Education Department) under MyRA Incentive Grant for $\mathrm{CO}_{2}$ Rich Natural Gas Value Chain Program are duly acknowledged.

\section{Reference}

[1] Report of U.S. Energy Information Administration. The International Energy Outlook; http://www.eia.gov/forecasts/ieo/ (accessed December, 2013).

[2] Scholes. C. A., Stevens. G. W.,Kentish. S. E., Membrane gas separation applications in natural gas processing. Fuel 96(2012)15-28.

[3] Zhang, Y., Sunarso. Liu. S., and Wang. R. Current status and development of membranes for $\mathrm{CO}_{2} / \mathrm{CH}_{4}$ separation: A review. Int J Greenh Gas Con 12 (2013) 84-107.

[4] Yeo. Z. Y., Chew. T. L, Zhu. P. W., Mohamed. A. R., Chai. S. P ., Conventional processes and membrane technology for carbon dioxide removal from natural gas- A review. J. Nat. Gas Chem.21(2012) 282-298.

[5] Pandey.P., Chauhan.R.S., Membranes for gas separation. Prog. Polym. Sci. 26(2001) 853-893.

[6] Yeo. Z. Y., Chew. T. L, Zhu. P. W., Mohamed. A. R., Chai. S. P ., Synthesis and performance of microporous inorganic membranes for $\mathrm{CO}_{2}$ separation-A review." J. Porous Mater. 20(6): 1457-1475.

[7] Himeno.S., Tomita.T, Suzuki. K, Nakayama. K, Yajima. K, Yoshida. S., Synthesis and Permeation Properties of a DDR3-Type Zeolite Membrane for Separation of $\mathrm{CO}_{2} / \mathrm{CH}_{4}$ Gaseous Mixtures. Ind. Eng. Chem. Res 46 (2007) 6989-6997. 
[8] Gucuyener. C., Van dan Bergh. J., Joaristi. A. M., Magusin. P. C. M. M,. Hensen. E. J . M., Gascon. J., Kapteijn. F., Facile synthesis of the DD3R zeolite: performance in the adsorptive separation of buta-, 3-diene and but-2-ene isomers. J. Mater. Chem., 21(2011) 18386-18397.

[9] Alves, M., A study of DDR3-type zeolite crystals and membranes (2007).

[10] Gies. H., Studies on clathrasils.9. crystal-structure of deca-dodecasil 3R, the missing link between zeolites and clathrasils, Z. Kristallogr. Zeitschrift fur Kristallographie, 175(1986) 93104.

[11] den Exter.M. J., Jansen. J. C., van Bekkum.H., Separation of Permanent Gases on the AllSilica 8-Ring Clathrasil DD3R, in: J.Weitkamp., H.G.Karge, W.Holderich., Studies in Surface Science and Catalysis. Elsevier Science.1994, 84:pp1159-pp1166.

[12] Tomita. T., Nakayama. K., Sakai.H., Gas separation characteristics of DDR3 type zeolite membrane. Microporous Mesoporous Mater 68(2004)71-75.

[13] Qi-Liang. Y., Sheng-Lai. Z., Xiao. L. Synthesis of DDR3-Type zeolite in fluoride medium. Chin. J. Inorg. Chem., 2(2009) 002.

[14] Sen. M., Bose. A., Pal. P., Das. J. K., Das. N. Rapid synthesis of DDR3 zeolite at room temperature. J. Am. Ceram. Soc., 97(2013) 52-55. 